\title{
New nonadiabatic pulsation computations on full PG 1159 evolutionary models: the theoretical GW Virginis instability strip revisited
}

\author{
A. H. Córsico ${ }^{1,2, \star}$, L. G. Althaus ${ }^{1,2, \star}$, and M. M. Miller Bertolami ${ }^{1,2,3, \star \star}$ \\ 1 Facultad de Ciencias Astronómicas y Geofísicas, Universidad Nacional de La Plata, Paseo del Bosque S/N, \\ (1900) La Plata, Argentina \\ e-mail: acorsico,althaus,mmiller@fcaglp.unlp.edu.ar \\ 2 Instituto de Astrofísica La Plata, IALP, CONICET-UNLP \\ 3 Max-Planck-Institut für Astrophysik, Garching, Germany
}

Received 12 April 2006 / Accepted 8 July 2006

\section{ABSTRACT}

\begin{abstract}
Aims. We reexamine the theoretical instability domain of pulsating PG 1159 stars (GW Vir variables).
Methods. We performed an extensive $g$-mode stability analysis on PG 1159 evolutionary models with stellar masses ranging from 0.530 to $0.741 M_{\odot}$, for which the complete evolutionary stages of their progenitors from the ZAMS, through the thermally pulsing AGB and born-again phases to the domain of the PG 1159 stars have been considered.

Results. We found that pulsations in PG 1159 stars are excited by the $\kappa$-mechanism due to partial ionization of carbon and oxygen, and that no composition gradients are needed between the surface layers and the driving region, much in agreement with previous studies. We show, for the first time, the existence of a red edge of the instability strip at high luminosities. We found that all of the GW Vir stars lay within our theoretical instability strip. Our results suggest a qualitative good agreement between the observed and the predicted ranges of unstable periods of individual stars. Finally, we found that generally the seismic masses (derived from the period spacing) of GW Vir stars are somewhat different from the masses suggested by evolutionary tracks coupled with spectroscopy. Improvements in the evolution during the thermally pulsing AGB phase and/or during the core helium burning stage and early AGB could help to alleviate the persisting discrepancies.
\end{abstract}

Key words. stars: evolution - stars: interiors - stars: oscillations - white dwarfs

\section{Background}

Pulsating PG 1159 stars - after the prototype of the spectral class and the variable type, PG 1159-035 or GW Vir - are very hot hydrogen-deficient post-Asymptotic Giant Branch (AGB) stars with surface layers rich in helium $(\sim 30-85 \%)$, carbon $(\sim 15-60 \%)$ and oxygen $(\sim 2-20 \%)$ (Werner \& Herwig 2006) that exhibit multiperiodic, low degree $(\ell \leq 2)$, high radial order $(k \gtrsim 18) g$-mode luminosity variations with periods in the range from about 300 to $3000 \mathrm{~s}$. Some GW Vir stars are still embedded in a planetary nebula; they are commonly called PNNVs (Planetary Nebula Nucleus Variable). PNNV stars are characterized by much higher luminosity than the "naked" GW Vir stars (those without nebulae $)^{1}$. GW Vir stars are particularly important to infer fundamental properties about pre-white dwarfs in general, such as the stellar mass and the surface compositional stratification $^{2}$ (Kawaler \& Bradley 1994; Córsico \& Althaus 2006).

^ Member of the Carrera del Investigador Científico y Tecnológico, CONICET, Argentina.

$\star \star$ Fellow of CONICET, Argentina.

${ }^{1}$ In this paper, naked GW Vir stars and PNNV stars will be indistinctly referred to as GW Vir stars or pulsating PG 1159.

${ }^{2}$ In addition, pulsating PG 1159 stars have recently been shown by Córsico \& Althaus (2005) to be valuable tools to constrain the occurrence of extra mixing episodes in their progenitor stars.
PG 1159 stars are believed to be the evolutionary connection between post-AGB stars and most of the hydrogen-deficient white dwarfs. These stars are thought to be the result of a born again episode triggered either by a very late helium thermal pulse (VLTP) occurring in a hot white dwarf shortly after hydrogen burning has almost ceased (see Fujimoto 1977; Schönberner 1979 and more recently Althaus et al. 2005) or a late helium thermal pulse (LTP) that takes place during the post-AGB evolution when hydrogen burning is still active (see Blöcker 2001, for references). During a VLTP episode, most of the hydrogen-rich envelope of the star is burnt in the helium-flash convection zone, whilst in a LTP hydrogen-deficient composition is the result of a dilution episode. In both cases, the star returns rapidly to the AGB and finally into the domain of high effective temperature as a hydrogen-deficient, quiescent helium-burning object.

A longstanding problem associated with pulsating PG 1159 stars is related to the excitation mechanism. The early work by Starrfield et al. (1983) was successful in finding the correct destabilizing agent, namely the $\kappa$-mechanism associated with the partial ionization of the K-shell electrons of carbon and/or oxygen in the envelope of models. However, their models required a driving region very poor in helium in order to be able to excite pulsations; even very low amounts of helium could weaken or completely remove the destabilizing effect of carbon and oxygen (i.e. "helium poisoning"). The latter requirement led to the conjecture that a composition gradient would exist to make 
compatible the helium-devoid driving regions and the heliumrich photospheric composition. Even modern detailed calculations still reveal the necessity of a compositional gradient in the envelopes of models (Bradley \& Dziembowski 1996; Cox 2003). The presence of a chemical composition gradient is difficult to explain in view of the fact that PG 1159 stars are still experiencing mass loss $\left(\dot{M} \sim 10^{-8.1} M_{\odot} \mathrm{yr}^{-1}\right.$ for PG 1159-035; Koesterke et al. 1998), a fact that prevents the action of gravitational settling of carbon and oxygen, and instead, tends to homogenize the envelope of hot white dwarfs (Unglaub \& Bues 2000).

Clearly at odds with the hypothesis of a composition gradient in the PG 1159 envelopes, calculations by Saio (1996), Gautschy (1997), and Quirion et al. (2004) - based on modern opacity OPAL data - demonstrated that $g$-mode pulsations in the correct ranges of effective temperatures and periods could be easily excited in PG 1159 models having a uniform envelope composition. The most recent study of PG 1159-type pulsations is that of Gautschy et al. (2005) (hereinafter GAS05) based on a full PG 1159 evolutionary sequence starting from the zero-age main sequence (ZAMS) and evolving through the thermally pulsing and VLTP phases (see Althaus et al. 2005). These authors found no need to invoke composition gradients in the PG 1159 envelopes to promote instability.

As important as they are, the vast majority of the studies of pulsation driving in PG 1159 stars rely on simplified stellar models. Indeed, the earliest works employed static envelope models and old opacity data. Even more modern works, although based on updated opacity data (OPAL), still use a series of static envelope models that do not represent a real evolutionary sequence, or evolutionary computations based on simplified descriptions of the evolution of their progenitors. The only exception is the work of GAS05, which employs equilibrium PG 1159 models that evolved through the AGB and born-again stages, beginning from a $2.7 M_{\odot}$ zero age main sequence model star. GAS05 analyzed four model sequences, with $0.530,0.55,0.589$ and $0.64 M_{\odot}$, the $0.589 M_{\odot}$ sequence being derived directly from the evolutionary computations of Althaus et al. (2005). The remaining sequences were created from the $0.589 M_{\odot}$ one by changing the stellar mass shortly after the end of the born-again episode.

On the basis of full evolutionary PG 1159 models covering the whole range of observed GW Vir masses, this paper aims to confirm and extend the results of the stability analysis by Saio (1996), Gautschy (1997), Quirion et al. (2004), and GAS05. We analyze the pulsational stability of seven different evolutionary sequences of PG 1159 models with stellar masses between 0.530 and $0.741 M_{\odot}$. Here, all of the PG 1159 evolutionary sequences have been derived by considering the complete evolution of their progenitors, an aspect that constitutes an improvement over previous studies. One of such sequences $\left(0.589 M_{\odot}\right)$ was presented by Althaus et al. (2005) and analyzed by GAS05, and the remaining ones were computed by Miller Bertolami \& Althaus (2006), with the exception of the $0.741 M_{\odot}$ sequence, which is presented for the first time in this work. The pulsational results presented here based on extensive full evolutionary models increase our understanding of the GW Vir stars and place previous studies on a solid basis, regarding stellar modeling. The paper is organized as follow: in the next section we briefly describe the input physics of our evolutionary code and the PG 1159 evolutionary sequences analyzed. A brief description of our nonadiabatic pulsation code is presented. In Sect. 3 we describe the stability analysis and in Sect. 4 we compare our predictions with the observed properties of known GW Vir stars. In Sect. 5 we summarize our main results and make some concluding remarks.
Table 1. Initial and final stellar mass (in solar units), and the final surface chemical abundances by mass (PG 1159 regime) for the evolutionary sequences considered in this work.

\begin{tabular}{llccccc}
\hline \hline$M_{\text {ZAMS }}$ & $M_{\text {PG }}$ & ${ }^{4} \mathrm{He}$ & ${ }^{12} \mathrm{C}$ & ${ }^{13} \mathrm{C}$ & ${ }^{14} \mathrm{~N}$ & ${ }^{16} \mathrm{O}$ \\
\hline 1 & 0.530 & 0.33 & 0.39 & 0.051 & 0.019 & 0.17 \\
1 & 0.542 & 0.28 & 0.41 & 0.051 & 0.018 & 0.21 \\
2.2 & 0.565 & 0.39 & 0.27 & 0.048 & 0.027 & 0.22 \\
2.7 & 0.589 & 0.31 & 0.38 & 0.040 & 0.012 & 0.23 \\
3.05 & 0.609 & 0.50 & 0.35 & 0.003 & 0.002 & 0.10 \\
3.5 & 0.664 & 0.47 & 0.33 & 0.019 & 0.013 & 0.13 \\
3.75 & 0.741 & 0.48 & 0.34 & 0.0007 & 0.0002 & 0.14 \\
\hline
\end{tabular}

\section{Evolutionary sequences}

The nonadiabatic pulsational analysis presented in this work relies on stellar models that take into account the complete evolution of the PG 1159 progenitor stars. The evolution of such models has been computed with the LPCODE evolutionary code, which is described in Althaus et al. (2005). LPCODE uses OPAL radiative opacities (including carbon- and oxygenrich mixtures) from the compilation of Iglesias \& Rogers (1996), complemented in the low-temperature regime with the molecular opacities of Alexander \& Ferguson (1994) (with solar metallicity). Chemical changes are performed via a time-dependent scheme that simultaneously treats nuclear evolution and mixing processes due to convection, salt fingers and overshooting. Convective overshooting is treated as an exponentially decaying diffusive process above and below any convective region.

Specifically, the background of stellar models has been extracted from the evolutionary calculations recently presented in Miller Bertolami \& Althaus (2006) and Althaus et al. (2005), who computed the full evolution of $1,2.2,2.7,3.05$, and $3.5 M_{\odot}$ stars. We include a new sequence of initially $3.75 M_{\odot}$. All of the sequences were evolved from the ZAMS through the thermally pulsing and mass loss phases on the AGB. After experiencing several thermal pulses, the progenitors depart from the AGB and evolve towards high effective temperatures. Mass loss during the departure from the AGB has been arbitrarily fixed so as to obtain a final helium shell flash during the early white dwarf cooling phase. After the born-again episode, the hydrogen-deficient, quiescent helium-burning remnants evolve at constant luminosity to the domain of PG 1159 stars with surface chemical composition rich in helium, carbon and oxygen. The masses of the remnants span the range $0.530-0.741 M_{\odot}$. For the sequence of $1 M_{\odot}$ two different AGB evolution have been considered, with different mass loss rates so as to obtain different numbers of thermal pulses and, eventually, two different remnant masses of 0.530 and $0.542 M_{\odot}$. The main characteristics of the sequences considered in this work are given in Table 1. We list the initial and final stellar mass (at the ZAMS and PG 1159 stages, respectively), and the surface abundance of the main chemical constituents during the PG 1159 stage. The sequence with an initial mass of $2.7 M_{\odot}$ is the same as presented by Althaus et al. (2005).

Our PG 1159 models are characterized by envelopes with uniform chemical compositions that extend from the surface downwards well below the driving region (i.e., the chemical composition at the driving region is the same as at the stellar surface). Thus, our models are not characterized by chemical gradients between the driving region and the stellar surface. Note that our post-VLTP models predict a range in the surface composition. In particular, the final surface abundance of 
helium spans the range $0.28-0.50$ by mass $^{3}$, which is in agreement with the range of observed helium abundance in most PG 1159s (see Werner \& Herwig 2006). Our sequences with helium abundances larger than the standard ones observed in PG 1159 stars will allow us to explore the role of helium in the instability properties of pulsating PG 1159s. Mass loss episodes after the VLTP have not been considered in the PG 1159 evolutionary sequences we employed here.

The pulsation stability analysis was performed with a new finite-difference nonadiabatic pulsation code based on the adiabatic version described in Córsico \& Althaus (2005, 2006). The nonadiabatic code solves the full sixth-order complex system of linearized equations and boundary conditions as given by Unno et al. (1989). Our code provides the dimensionless complex eigenvalue $(\omega)$ and eigenfunctions $\left(y_{1}, \ldots, y_{6}\right)$ as given by Unno et al. (1989). Nonadiabatic pulsation periods and normalized growth rates are evaluated as $\Pi=2 \pi / \mathfrak{R}(\sigma)$ and $\eta=$ $-\mathfrak{J}(\sigma) / \mathfrak{R}(\sigma)$, respectively. Here, $\mathfrak{R}(\sigma)$ and $\mathfrak{J}(\sigma)$ are the real and the imaginary part, respectively, of the complex eigenfrequency $\sigma=\left(G M_{*} / R_{*}^{3}\right)^{1 / 2} \omega$. Our code also computes the differential work function, $\mathrm{d} W(r) / \mathrm{d} r$, and the running work integral, $W(r)$, as in Lee \& Bradley (1993). In this work the "frozen-in convection" approximation was assumed because the flux carried by convection is usually negligible in PG 1159 stars. Also, the $\epsilon$-mechanism for mode driving was neglected in the computations because nuclear-burning shells in PG 1159 models usually destabilize very short periods that are not observed in GW Vir stars (Kawaler et al. 1986; Kawaler 1988; Gautschy 1997). We employed about 3000 mesh-points to describe our background stellar models, most of them distributed in the envelope region where all the pulsation driving and damping occur. We employed the "Ledoux modified" treatment to compute the Brunt-Väisälä frequency $(N)$ (Tassoul et al. 1990).

\section{Stability calculations}

We analyze the stability properties of about 2400 stellar models covering a wide range of effective temperatures $(5.4 \gtrsim$ $\left.\log \left(T_{\text {eff }}\right) \gtrsim 4.8\right)$ and a range of stellar masses $(0.530 \lesssim$ $\left.M_{*} / M_{\odot} \lesssim 0.741\right)$. For each model we have restricted our study to unstable $\ell=1,2 g$-modes with periods in the range $50 \mathrm{~s} \lesssim \Pi \lesssim$ $7000 \mathrm{~s}$, thus comfortably covering the full period spectrum observed in GW Vir stars. Consistent with other stability studies of GW Vir stars, all unstable $g$-modes in our PG 1159 models are driven by the $\kappa$-mechanism associated with the opacity bump due to partial ionization of $\mathrm{K}$-shell electrons of $\mathrm{C}_{\mathrm{V}-\mathrm{VI}}$ and $\mathrm{O}_{\mathrm{VII}-\mathrm{VIII}}$ centered at $\log T \approx 6.2$ (Quirion et al. 2004; GAS05).

We start by discussing the stability properties of two template $0.589 M_{\odot}$ PG 1159 models. These properties are common to all PG 1159 models of our complete set of evolutionary sequences. The normalized growth rate $(\eta)$ in terms of pulsation periods $(\Pi)$ for overstable $\ell=1$ modes corresponding to the two selected models are shown in Fig. 1. Model (a) is representative of the high-luminosity, low-gravity pre-white dwarf regime, and model (b) is typical of the low-luminosity, high-gravity phase, when the object has already entered their white dwarf cooling track (see Fig. 3). Note that modes excited in model (a)

3 These abundances are not only determined by the stellar mass, but also by the deepness of the envelope convection and mass loss episodes after the VLTP (see Miller Bertolami \& Althaus 2006 for comments). Also, the number of thermal pulses considered in the AGB stage and the efficiency of overshooting play a role in the final PG 1159 surface composition (Herwig 2000).

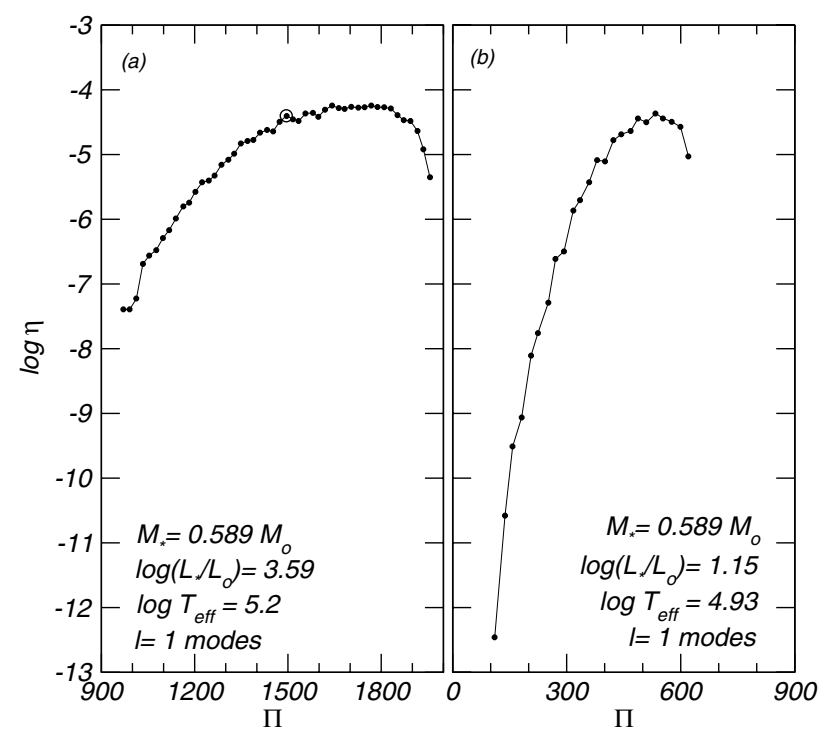

Fig. 1. The normalized growth rate in terms of period (in seconds) for overstable $g$-modes corresponding to two $0.589 M_{\odot}$ PG 1159 models. The location of these models on the HR diagram is shown in Fig. 3. The circumscribed dot in panel a) corresponds to a mode with $k=70$.

have pulsation periods in the range $1000 \lesssim \Pi \lesssim 2000 \mathrm{~s}$, substantially longer than those excited in model (b) $(100 \lesssim \Pi \lesssim 600 \mathrm{~s})$. For each model, $\eta$ reaches a maximum value in the vicinity of the long-period boundary of the instability domain. In other words, within a given band of unstable modes, the excitation is markedly stronger for modes characterized by long periods. This effect is particularly strong in model (b), the value of the growth rate for the shortest periods being more than seven orders of magnitude smaller than for the modes with longer periods ${ }^{4}$.

Figure 2 shows details of the driving/damping process in model (a) for two selected dipole modes. We restrict the figure to the envelope region of the model, where the main driving and damping occurs. Thick continuous curve corresponds to $\mathrm{d} W / \mathrm{d} r$ for an unstable mode with $k=70, \Pi=1496 \mathrm{~s}$ and $\eta=3.9 \times$ $10^{-5}$ (marked as a circumscribed dot in panel (a) of Fig. 1), while the thick dashed curve depicts the situation for a stable mode with $k=150, \Pi=3216 \mathrm{~s}, \eta=-1.23 \times 10^{-3}$. Also plotted is the Rosseland opacity, $\kappa$, and its logarithmic derivatives, $\kappa_{\mathrm{T}}$ and $\kappa_{\rho} /\left(\Gamma_{3}-1\right)$. The region that destabilizes the $k=70$ mode (where $\mathrm{d} W / \mathrm{d} r>0$ ) is clearly associated with the bump in the opacity centered at $-\log q \approx 8.4\left[q \equiv\left(1-M_{r} / M_{*}\right)\right]$, although the maximum driving for this mode comes from a slightly more internal region $(-\log q \approx 8)$. Note also that in the driving region the quantity $\kappa_{\mathrm{T}}+\kappa_{\rho} /\left(\Gamma_{3}-1\right)$ is increasing outward, in agreement with the well known necessary condition for mode excitation (Unno et al. 1989). Since the contributions to driving at $-\log q$ from 7.5 to 10 largely overcome the damping effects at $6 \lesssim-\log q \lesssim 7.5$, the mode with $k=70$ is globally excited. At variance, the strong damping experienced by the mode with $k=150$ (denoted by negative values of $\mathrm{d} W / \mathrm{d} r$ ) makes this mode globally stable. The situation in the low-luminosity, high-gravity phase as in model (b) of Fig. 1 is similar, the only important difference being that the driving/damping regions are located in considerably more external layers. This is due to an outward migration of the opacity profile, induced by the evolution of the star.

\footnotetext{
4 We note, however, that for modes with the shortest periods the value of $\eta$ is so small $\left(\lesssim 10^{-9}\right)$ that they are only marginally unstable.
} 


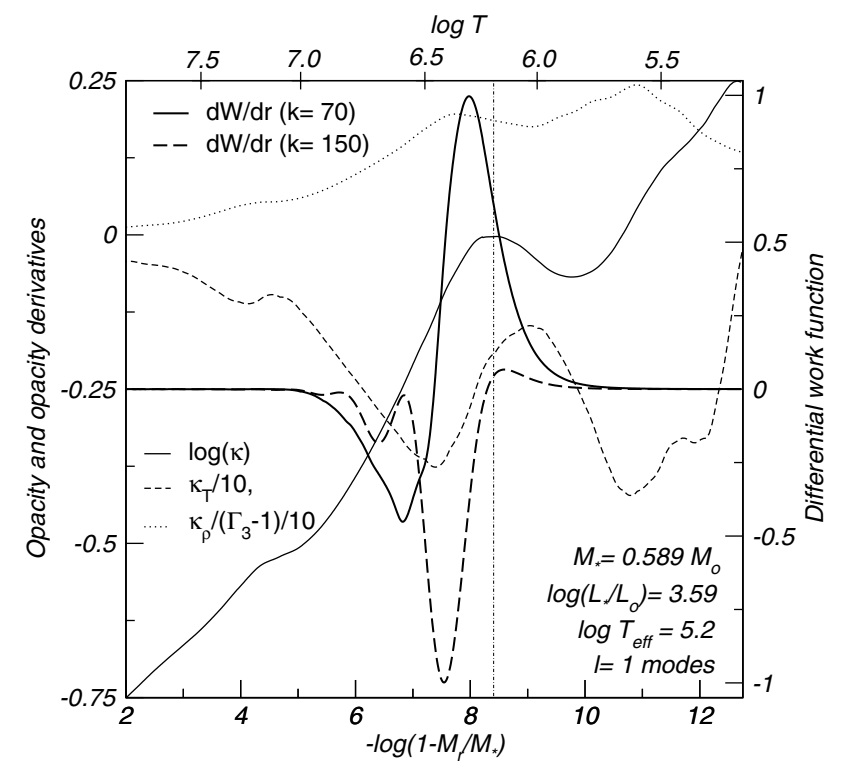

Fig. 2. The opacity and opacity derivatives (left scale), and the differential work function (right scale) for two selected $\ell=1$ modes in terms of the mass coordinate (lower scale) and the logarithm of the temperature (upper scale), corresponding to the PG 1159 model (a) analyzed in Fig. $1 . \mathrm{d} W / \mathrm{d} r$ for the mode with $k=70(k=150)$ is normalized to an extremum value of $+1(-1)$. The vertical dot-dashed line shows the location of the maximum in the $\mathrm{C} / \mathrm{O}$ opacity bump.

\subsection{The theoretical GW Vir instability strip}

Here, we examine the location of the unstable domains on the HR diagram. In Fig. 3 we show the evolutionary tracks for our complete set of PG 1159 model sequences, where the thick portions of the curves correspond to models with dipole unstable modes. A well-defined instability domain, bounded by a red (cool) edge at high luminosities, and by a blue (hot) edge both at high and low luminosities, is apparent in the plot. The blue and red edges for dipole and quadrupole modes for each sequence are connected by thin curves as given by standard nonlinear least-squares algorithms. The instability domains for $\ell=1$ and $\ell=2$ look very similar, although the edges for $\ell=2$ are slightly shifted to higher effective temperatures, and the region of instability is somewhat wider than for $\ell=1$. Figure 3 should be compared with Fig. 5 of GAS05. The global agreement between our results and the predictions of GAS05 is excellent, in particular for the sequence of $0.589 M_{\odot}$ - the only sequence in common between those authors and our work.

At variance with GAS05, in this work we have employed PG 1159 models with different masses derived from the complete evolution of the progenitor stars. This has enabled us to extend the pulsational stability analysis to lower effective temperatures in the high-luminosity, low-gravity region. As a result, we have found, for the first time, a reliable high-luminosity, lowgravity red edge of the GW Vir instability strip. The red edge is markedly sensitive to the stellar mass, being hotter for the more massive models.

Our blue edge (both for dipole and quadrupole modes) cannot be exactly represented by a straight line. It is clearly seen for dipole modes in Fig. 3. The departures from a simple linear relation have their origin in the different surface chemical compositions with which our models of different stellar masses reach the domain of the PG 1159 after emerging from the bornagain episode. The unstable portions of the evolutionary tracks corresponding to models characterized by a surface helium

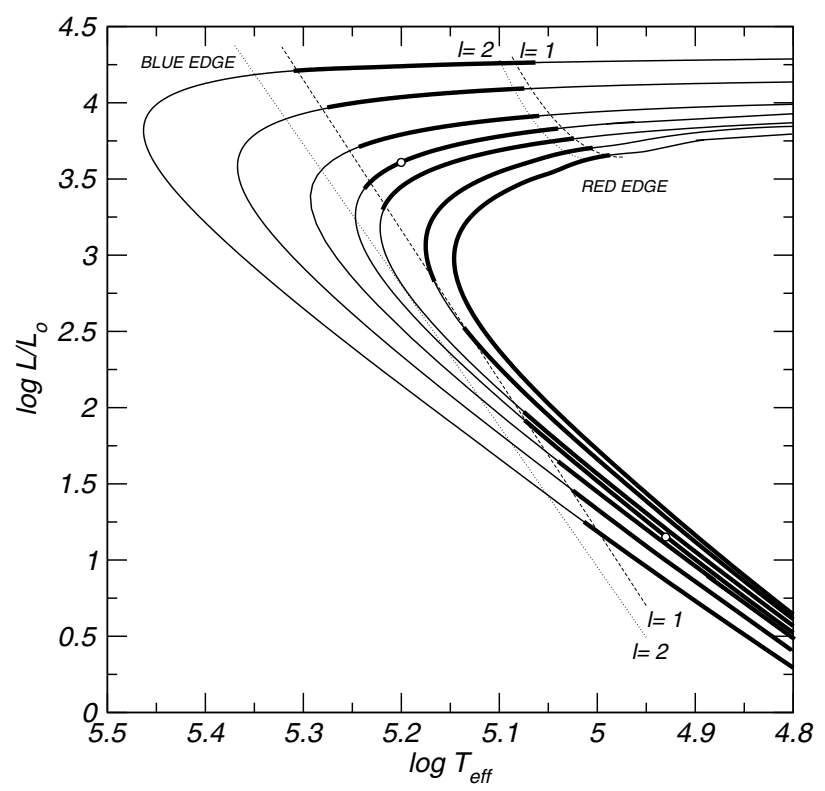

Fig. 3. The HR diagram for our complete set of PG 1159 model sequences of $0.530,0.542,0.565,0.589,0.609,0.664$ and $0.741 M_{\odot}$ (from right to left). The portions of the tracks harbouring stellar models with overstable $\ell=1 \mathrm{~g}$-modes are emphasized with thick solid lines. Thin dashed $(\ell=1)$ and dotted $(\ell=2)$ lines are parameterizations of the blue and red edges of instability. The small white circles on the $0.589 M_{\odot}$ track show the loci of the two template models analyzed in Fig. 1.

abundance of $\approx 0.28-0.39$ by mass (that is, the sequences with masses of $0.542,0.565$ and $0.589 M_{\odot}$ ) extend slightly beyond the linear parameterization of the blue edge, as compared with the case of the more massive models which have greater helium abundances in the envelope $(0.50,0.47$ and 0.48 for stellar masses of $0.609,0.664$ and $0.741 M_{\odot}$, respectively) ${ }^{5}$. Increasing the helium abundance in the driving region, the efficiency of pulsational driving is reduced (see GAS05). This is consistent with the finding of Quirion et al. (2004) that decreasing the helium mass fraction in the driving regions, the blue edge of the instability domain shifts to higher effective temperatures.

Since spectroscopic calibration of PG 1159 stars gives effective temperatures and surface gravities, we plot the evolutionary tracks and the instability domains on the $\log \left(T_{\text {eff }}\right)-\log g$ plane. Figure 4 gives the complete set of PG 1159 sequences. We emphasize with thick solid lines the stages with overstable $\ell=1$ $g$-modes and the loci of the blue and red edges for dipole and quadrupole modes with thin curves as parameterized by standard nonlinear least-squares procedures. We postpone to Sect. 4 a complete discussion of the general agreement between our pulsation models and the observed GW Vir stars.

In the current computations, the dipole unstable domain for each evolutionary sequence is separated into two regions, one of them corresponding to the high-luminosity phase (low gravity) and the other corresponding to low luminosities (high gravity) (see Figs. 3 and 4). The only exception is the sequence with $0.530 M_{\odot}$ which exhibits an unique unstable domain that extends uninterruptedly from the high-luminosity phase to the lowluminosity regime. This is at variance with the results reported by GAS05, who found that for all of their sequences (including that with $0.530 M_{\odot}$ ) the tip of the evolutionary "knee" is

\footnotetext{
${ }^{5}$ Note that the helium abundance range predicted by our models (0.3-0.5) is coincident with the observed one for most of PG 1159 stars, as reported by Werner \& Herwig (2006).
} 


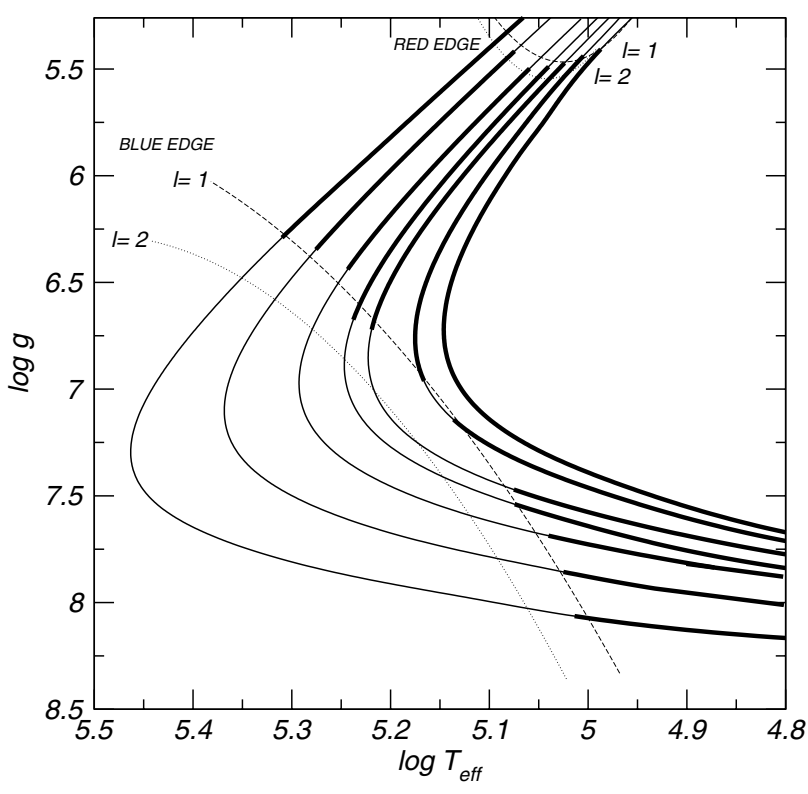

Fig. 4. Same as Fig. 3, but for the $\log \left(T_{\text {eff }}\right)-\log g$ plane.

pulsationally stable in the case of dipole modes. This discrepancy is related to the different loci of the tracks on the HR diagram and on the $\log \left(T_{\text {eff }}\right)-\log g$ plane. The evolutionary sequences considered in this work reach lower $\log g$ and luminosity values for a fixed $\log \left(T_{\text {eff }}\right)$ than in the ones of GAS05. This reflects the fact that in the present work we have considered PG 1159 evolutionary sequences derived from the full evolutionary computations of the progenitor stars.

We now explore the ranges of periods of unstable modes. Figure 5 shows the instability boundaries of dipole modes on the $\log \left(T_{\text {eff }}\right)-\Pi$ plane for the complete set of evolutionary sequences. Note that generally the periods of unstable modes for each sequence are clearly grouped in two separated regions, one of them characterized by long periods and high-radial overtones, corresponding to evolutionary stages before the knee at high luminosities, and the other one characterized by short periods (low $k$ values) corresponding to the hot portion of the white dwarf cooling track (low luminosities). As mentioned before, the sequence with $0.530 M_{\odot}$ shows a unique instability domain. Thus, this sequence shows instability even around the knee. The splitting of the instability domains into two separate regions can be understood in terms of the magnitude of the thermal timescale at the driving region. See GAS05 for a demonstration of this.

The high-luminosity domain of instability exhibits a strong mass dependence, the longest unstable periods being shorter for the more massive models. In addition, for the less massive models the longest pulsation period is reached at lower effective temperatures the more massive models. In all the cases the long-period limit is attained shortly after the beginning of the instability domain. The shortest unstable period for each sequence is also markedly sensitive to the stellar mass. Generally, the minimum period is smaller for the less massive models. Thus, the period-width of the instability domain is larger for the less massive models. As can be seen from Fig. 5, the instability island on the $\log \left(T_{\text {eff }}\right)-\Pi$ plane is almost vertical for the model with $0.530 M_{\odot}$, but its slope gradually decreases as we go to sequences with higher masses.

The low-luminosity domain, on the other hand, shows a moderate dependence on the stellar mass. The maximum overstable period is always larger for the less massive models. The

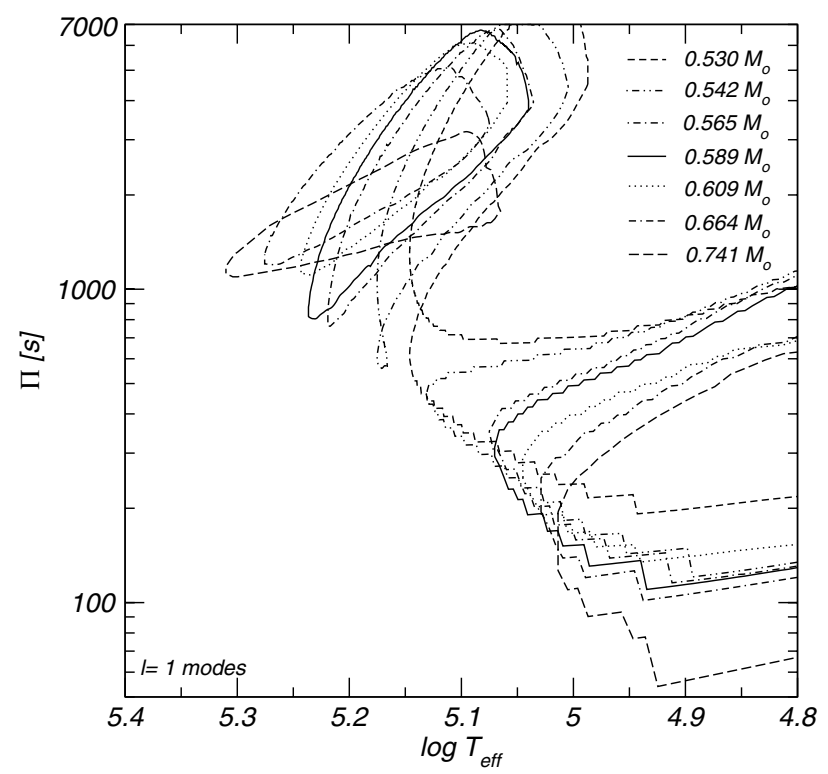

Fig. 5. The instability domains on the $\log \left(T_{\text {eff }}\right)-\Pi$ plane for $g$-modes with $\ell=1$ corresponding to our complete set of PG 1159 model sequences. Different line styles depict the situation for different stellar masses.

minimum overstable period, however, does not show a clear trend with the value of the stellar mass. We note that the shortest unstable periods are of $\approx 55 \mathrm{~s}$ and correspond to the sequence with $0.741 M_{\odot}$. This short-period limit is substantially lower than that reported by GAS05, of about $190 \mathrm{~s}$ for their sequence of $0.64 M_{\odot}$. In our computations the shortest overstable periods have very small growth rates, of the order of $10^{-9}-10^{-13}$, and thus we could consider that these modes are stable (see panel (b) of Fig. 1). If so, our short-period limit agrees with the predictions of GAS05. The morphology of the instability domains for quadrupole modes looks very similar to the case of dipole modes, the novel feature being a shortening of the overstable periods, in agreement with GAS05. Indeed, the long-period limits of the high-luminosity instability domains for quadrupole modes are shortened by about $3000 \mathrm{~s}$ relative to dipole ones. A less severe decreasing of overstable quadrupole periods is also present in the low-luminosity domain. As a result, both regions of instability are closer together to such a degree that the high- and lowluminosity instablility domains of the $0.530,0.542$ and $0.565 M_{\odot}$ sequences merge into an uninterrupted region ${ }^{6}$. The lowest $\ell=2$ short-period limit for the low-luminosity domain, which corresponds to the sequence with $M_{*}=0.741 M_{\odot}$, is of about $50 \mathrm{~s}$.

In all of the computations presented in this paper we neglected chemical diffusion, and thus the stability calculations were performed on PG 1159 models with a constant chemical composition in the driving region. In particular, no helium enrichment in the driving region was allowed, and consequently all of our sequences shown pulsational instability well beyond the empirical red edge of the GW Vir stars at low luminosities $\left(T_{\text {eff }} \sim 75000 \mathrm{~K}\right.$; Dreizler \& Heber 1998), even down to the domain of the variable DB white dwarfs.

${ }^{6}$ Note that in Figs. 3 and 4 the blue edge for $\ell=2$ crosses the evolutionary track of $0.565 M_{\odot}$; it is a parameterization of the blue edge and not the exact theoretical blue edge emerging from our stability analysis. 
Table 2. Stellar parameters and pulsation properties of all known pulsating PG 1159 stars. The approximate surface abundances (in \% by mass) have been derived from Table 2 of Werner \& Herwig (2006) by assuming a composition made of ${ }^{4} \mathrm{He},{ }^{12} \mathrm{C}$ and ${ }^{16} \mathrm{O}$, except for $\mathrm{HS} 2324+3944$ (data taken from Table 1 of Werner \& Herwig 2006) and for Abell 43 (data taken from Miksa et al. 2002).

\begin{tabular}{rccccccccc}
\hline \hline Star & $\begin{array}{c}\log \left(T_{\text {eff }}\right)^{a} \\
{[\mathrm{~K}]}\end{array}$ & $\begin{array}{c}\log g^{a} \\
{[\mathrm{cgs}]}\end{array}$ & $\begin{array}{c}M_{*}^{b} \\
{\left[M_{\odot}\right]}\end{array}$ & H & He & C & O & Remark & $\begin{array}{c}\text { Observed period range }(\ell=1) \\
{[\mathrm{s}]}\end{array}$ \\
\hline Abell 43 & 5.041 & 5.7 & 0.53 & 35 & 42 & 23 & - & PNNV, hybrid & $2600-3035^{c}$ \\
HS 2324+3944 & 5.114 & 6.2 & 0.53 & 17 & 35 & 42 & 6 & naked, hybrid & $2005-2569^{d}$ \\
Longmore 4 & 5.079 & 5.5 & 0.63 & - & 48 & 43 & 9 & PNNV & $831-2325^{e}$ \\
NGC 246 & 5.176 & 5.7 & 0.75 & - & 63 & 31 & 6 & PNNV & $1460-1840^{f}$ \\
K 1-16 & 5.146 & 6.4 & 0.54 & - & 33 & 50 & 17 & PNNV & $1500-1700^{g}$ \\
RX J2117+3412 & 5.230 & 6.0 & 0.72 & - & 39 & 55 & 6 & PNNV & $694-1530^{h}$ \\
HE 1429-1209 & 5.204 & 6.0 & 0.66 & - & 39 & 55 & 6 & naked & $919^{i}$ \\
PG 1159-035 & 5.146 & 7.0 & 0.54 & - & 33 & 50 & 17 & naked & $430-840^{j}$ \\
PG 2131+066 & 4.978 & 7.5 & 0.55 & - & 44 & 39 & 17 & naked & $339-598^{k}$ \\
PG 1707+427 & 4.929 & 7.5 & 0.53 & - & 44 & 39 & 17 & naked & $335-909^{l}$ \\
PG 0122+200 & 4.903 & 7.5 & 0.53 & - & 44 & 39 & 17 & naked & $336-612^{m}$ \\
\hline
\end{tabular}

References: ${ }^{a}$ Werner \& Herwig (2006); ${ }^{b}$ Miller Bertolami \& Althaus (2006); ${ }^{c}$ Vauclair et al. (2005); ${ }^{d}$ Silvotti et al. (1999); ${ }^{e}$ Bond \& Meakes (1990); ${ }^{f}$ Ciardullo \& Bond (1996); ${ }^{g}$ Grauer et al. (1987); ${ }^{h}$ Vauclair et al. (2002); ${ }^{i}$ Nagel \& Werner (2004); ${ }^{j}$ Winget et al. (1991); ${ }^{k}$ Kawaler et al. (1995); ${ }^{l}$ Kawaler et al. (2004); ${ }^{m}$ O’Brien et al. (1998).

\section{Theory vs. observations}

In this section we compare our theoretical predictions with the observed properties of GW Vir stars. Currently, 11 pulsating PG 1159 stars are known. In Table 2 we show the main spectroscopic and pulsation data available. Note that there are five GW Vir stars (termed PNNV) that are still embedded in a planetary nebula. The remaining objects lack a surrounding nebula and are commonly called "naked" GW Vir stars. There are two objects with measurable amounts of hydrogen in their spectra; they are termed pulsating "hybrid-PG 1159". HE 1429-1209 is a naked GW Vir star but its effective temperature and gravity place it in the region of the HR diagram usually populated by PNNVs.

In Fig. 6 we plot the location of pulsating and nonpulsating PG 1159 stars, as well as PG 1159 stars that have not been observed for variability, on the $\log \left(T_{\text {eff }}\right)-\log g$ plane (data taken from Werner \& Herwig 2006). The plot also shows the evolutionary tracks for our complete set of sequences of PG 1159 models. The blue and red edges for dipole and quadrupole modes are also shown. Regarding pulsating PG 1159 stars, the agreement between observations and model predictions is excellent. All of the GW Vir variables lie inside our predicted instability domains of dipole and quadrupole modes. However, there are several non-variables occupying the unstable region. The existence of non-variable stars within the instability domain could in part be understood in terms of a variation in surface chemical composition (and thus in the driving region) from star to star. For instance, Quirion et al. (2004) found that the helium enrichment in the driving region is the cause of the nonpulsator MCT 0130-1937 (with a helium abundance of about 75\%) within the instability strip. Note, however, that PG 1151-029, Longmore 3, Abell 21 and VV47 (not included in the analysis of Quirion et al. 2004) are found to have standard helium abundances (see Table 2 of Werner \& Herwig 2006) and however are non-variables. On the other hand, it is remarkable the existence of the pulsating star NGC 246 with a helium abundance $\left(X_{\mathrm{He}} \approx 0.63\right)$ unusually large among pulsators (see Table 2 ). These controversial cases remain to be explained.

Regarding PG 1159-035 (GW Vir) - the prototype of both the variable and the spectroscopic classes - our analysis predicts that this star pulsates for a stellar mass of $\sim 0.536 M_{\odot}$ (see Fig. 6). It is a markedly higher value than the mass required by GAS05 to excite pulsations in PG $1159-035\left(M_{*} \approx 0.50-0.51 M_{\odot}\right)$, and, at the same time, closer to the long-recognized "seismic" mass of $\approx 0.59 M_{\odot}$ of Kawaler \& Bradley (1994). So, the employment of PG 1159 models derived from the complete evolution of their progenitor stars appears to be a key factor to alleviate the discrepancy between the seismic and the spectroscopic mass of PG 1159-035.

In what follows we focus on the observed period ranges for the known GW Vir stars. In Fig. 7 we show the $\log \left(T_{\text {eff }}\right)-\Pi$ diagram, in which the effective temperatures and the period ranges are taken from Table 2. For comparison, we have included the theoretical instability boundaries for $\ell=1$. Also plotted are the ranges of unstable periods as predicted by Quirion et al. (2004). We are only interested in a qualitative comparison between the observed and the theoretical ranges of unstable periods. Thus, we do not attempt a detailed asteroseismic period fitting for each individual GW Vir star. As can be seen from Fig. 7, the agreement between theory and observations is reasonably good for all cases, and for several stars the agreement is excellent. Note, for instance, that for seven stars (Abell 43, HS 2324+3944, K1-16, NGC 246, HE 1429-1209, PG 2131+066 and PG 0122+200) the observed ranges of periods are completely contained in the theoretical instability domains corresponding to at least one stellar mass. For the case of PG 1159-035, the observed periods are compatible with the instability domain of the $0.53 M_{\odot}$ models, although the shorter detected periods $(\$ 530 \mathrm{~s})$ lie below the theoretical short-period limit. The opposite is true for PG $1707+427$, whose longer observed periods ( $2760 \mathrm{~s}$ ) lie above the highest long-period limit which corresponds, at the effective temperature of this star, to the $0.530 M_{\odot}$ sequence. In the case of RX J2117+3412, the range of observed periods partially overlaps with four instability regions, corresponding to the sequences of $0.5890,0.609,0.664$ and $0.741 M_{\odot}$, but no instability domain accounts for the observed periods shorter than $\approx 800 \mathrm{~s}$. Finally, in the case of Longmore 4 the band of observed periods is partially covered by the instability domains of the $0.530,0.542$ and $0.741 M_{\odot}$ sequences. Note that the agreement between the observed and predicted periods of unstable modes is comparable to that reported by Quirion et al. (2004).

The predicted instability domains resulting from our analysis nearly reproduce the observational trend that the periods exhibited by pulsating PG 1159 stars decrease with decreasing 


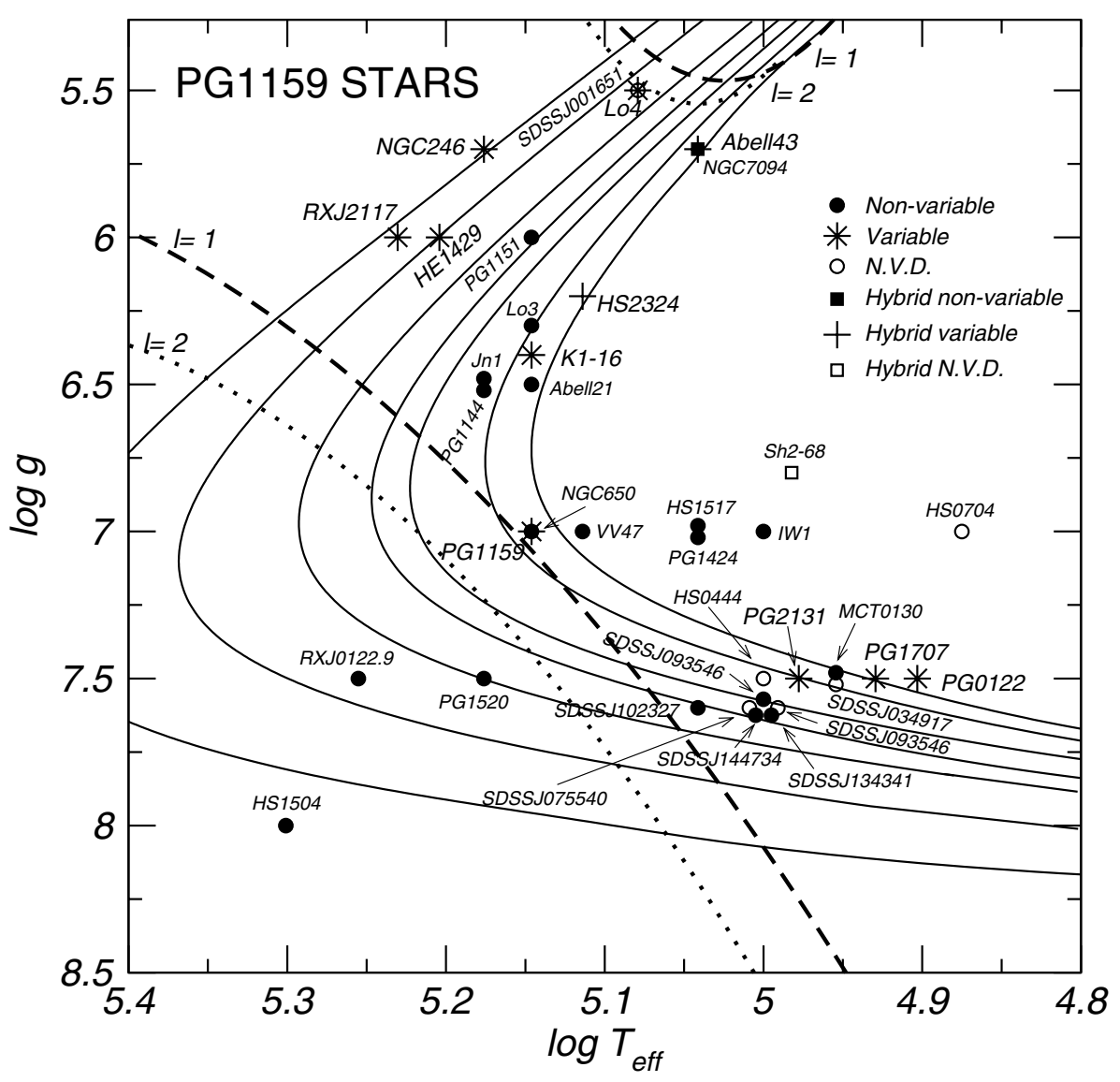

Fig. 6. The distribution of the spectroscopically calibrated variable and non-variable PG 1159 stars in the $\log \left(T_{\text {eff }}\right)-\log g$ plane. PG 1159 stars with no variability data (N.V.D.) are depicted with hollow symbols. Solid curves show the evolutionary tracks for different stellar masses: $0.530,0.542,0.565,0.589,0.609,0.664$ and $0.741 M_{\odot}$. Parameterizations of the theoretical dipole (dashed curves) and quadrupole (dotted curves) red and blue edges of the instability domain are also displayed.

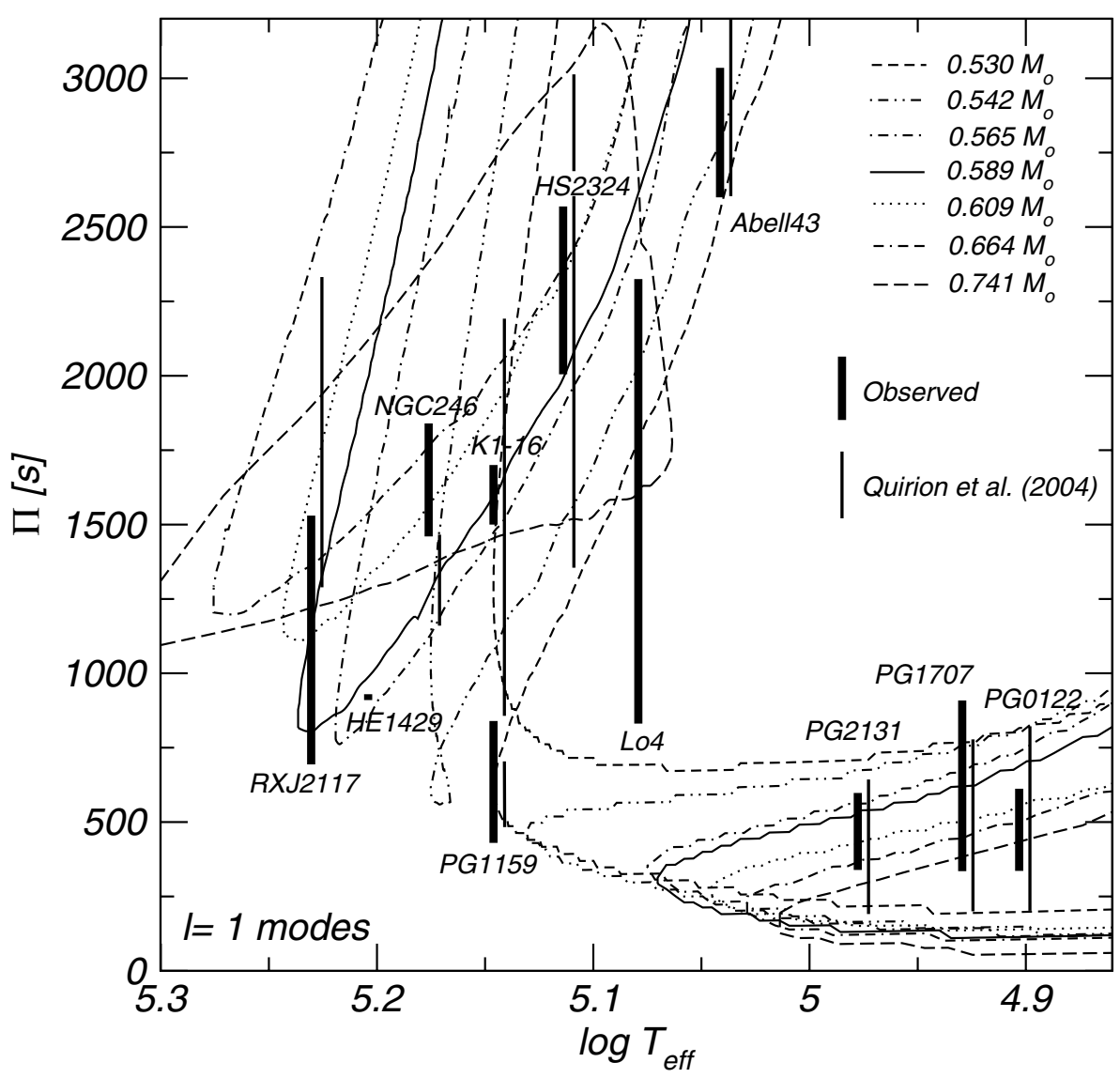

Fig. 7. The period ranges of known variable PG 1159 stars on the $\log \left(T_{\text {eff }}\right)-\Pi$ plane, depicted with thick vertical bars (the thickness of the bars is arbitrary). For comparison, we include the predicted ranges of unstable periods according to Quirion et al. (2004), shown with thinner vertical bars (arbitrarily shifted to the right for clarity). The theoretical instability domains for $g$-modes with $\ell=1$ for our complete set of PG 1159 sequences are depicted with different line styles. 
luminosity (increasing surface gravity) (see O'Brien 2000, for a discussion of such a trend). This is well documented by Figs. 5 and 7. Note that Longmore 4, being a high-luminosity PNNV that shows short periods, is an exception to this trend. Longmore 4 is particularly interesting because it showed a surprising behaviour in its spectral type which suddenly changed from PG 1159 to [WCE] and back to PG 1159. According to Werner et al. (1992), this could be the result of a transient but significant increase in the mass loss rate. According to our computations, Longmore 4 is located very close to the red edge of the instability strip (see Fig. 6); so, this star could have just entered the instability phase.

In addition to the issue of pulsation instability, valuable information about pulsating PG 1159 stars can be extracted from the period spacing between consecutive overtones of fixed $\ell$ value. The period spacing depends primarily on the stellar mass and is only weakly dependent on the luminosity and surface compositional stratification (Kawaler \& Bradley 1994; Córsico \& Althaus 2006). Thus, this quantity allows a determination of $M_{*}$ to a very high accuracy. Here, we consider five GW Vir stars for which detailed asteroseismic studies have

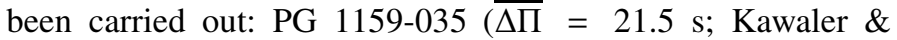
Bradley 1994), PG $2131+066(\overline{\Delta \Pi}=21.6 \mathrm{~s}$; Reed et al. 2000), PG $1707+427(\overline{\Delta \Pi}=23.0 \mathrm{~s} ;$ Kawaler et al. 2004), PG 0122+200 $(\overline{\Delta \Pi}=21.1 \mathrm{~s}$; O'Brien et al. 2000), and RX J2117+3412 $(\overline{\Delta \Pi}=21.5 \mathrm{~s}$; Vauclair et al. 2002). We compare the observed mean period spacing of each star with the average of the computed period spacing, $\overline{\Delta \Pi_{k}}\left(\Delta \Pi_{k}=\Pi_{k+1}-\Pi_{k}\right)$, corresponding to models with an effective temperature as close as possible to the value of $T_{\text {eff }}$ of the star under consideration.

We first consider the case of PG 1159-035. According to its location on the $\log \left(T_{\text {eff }}\right)-\log g$ plane, this star should have a stellar mass of $\approx 0.536 M_{\odot}$. We refer this value as the "spectroscopic mass", $M_{\text {spec }}$ (see Fig. 6). Our stability analysis predicts that a model with this mass at the effective temperature of PG 1159-035 is pulsationally unstable. However, this model should have a value of $\overline{\Delta \Pi}_{k} \approx 23 \mathrm{~s}$, which is in conflict with the observed mean value of $21.5 \mathrm{~s}$. To have a $\overline{\Delta \Pi_{k}}$ value compatible with the observed period spacing the stellar mass of PG $1159-035$ should be $M_{\text {seis }} \approx 0.558 M_{\odot}$. Thus, we found a discrepancy of $\Delta M_{*}=M_{\text {seis }}-M_{\text {spec }} \approx 0.022 M_{\odot}$, somewhat lower than that reported in the literature $\left(M_{\text {spec }} \approx 0.54 M_{\odot}\right.$, Dreizler $\&$ Heber 1998; $M_{\text {seis }} \sim 0.59 M_{\odot}$, Kawaler \& Bradley 1994). We note that a model with $M_{*} \approx 0.558 M_{\odot}$ at $140000 \mathrm{~K}$ should be pulsationally stable in the framework of our stability analysis.

In the case of RX J2117+3412, the evolutionary tracks of Fig. 6 suggest a stellar mass of about $0.72 M_{\odot}$ (see Miller Bertolami \& Althaus 2006). A model with this mass and at the $T_{\text {eff }}$ of this star should be pulsationally unstable. The corresponding value of $\overline{\Delta \Pi_{k}}$ should be lower than $16 \mathrm{~s}$, clearly at odds with the measured mean value of $21.5 \mathrm{~s}$. So, in order to have $\overline{\Delta \Pi_{k}}$ values comparable to the observed, we should be forced to consider models with masses lower than $0.57 M_{\odot}$. Thus, for RX J2117+3412 we found a large disagreement between the spectroscopic and the seismic mass $\left(\Delta M_{*} \approx-0.15 M_{\odot}\right)$ and in the opposite direction than for PG 1159-035. Note that models with such low masses (at the effective temperature of RX J2117+3412) are outside of the instability domain (see Fig. 6).

Finally, we have the cases of PG $2131+066$, PG 1707+427 and PG $0122+200$. According to our evolutionary tracks, the mass of these stars should be of $\approx 0.55 M_{\odot}$ for PG $2131+066$ and of $\approx 0.53 M_{\odot}$ for PG $1707+427$ and PG $0122+200$. However, in order to have values of $\overline{\Delta \Pi}_{k}$ compatible with the observed mean period spacings, the stellar masses should be substantially larger, of about $0.58 M_{\odot}$ for PG $2131+066\left(\Delta M_{*} \approx 0.03 M_{\odot}\right)$, $0.57 M_{\odot}$ for PG $1707+427\left(\Delta M_{*} \approx 0.04 M_{\odot}\right)$, and $0.65 M_{\odot}$ for PG $0122+200\left(\Delta M_{*} \approx 0.12 M_{\odot}\right)$. Models with such high stellar masses (at the effective temperature of the stars under consideration) are pulsationally unstable (see Fig. 6). Given the spectroscopic uncertainties in the determination of $\log g$, these solutions still could be compatible with those derived from our evolutionary tracks and stability analysis.

We conclude that the stellar masses of naked GW Vir stars as predicted by our evolutionary tracks are generally 4-20\% lower than those suggested by the period spacing data. On the contrary, for the PNNV RX J2117+3412 the evolutionary tracks predict a spectroscopic mass about $25 \%$ higher than the seismic derivation. Although our full evolutionary PG 1159 models hint at a general agreement between the spectroscopic and seismic masses of pulsating PG 1159 stars, persisting discrepancies could still reflect a problem in the stellar modelling during the pulsing AGB phase of progenitor stars, as noted by Werner \& Herwig (2006).

\section{Summary and conclusions}

In this paper we re-examined the pulsational stability properties of GW Vir stars. We performed extensive nonadiabatic computations on PG 1159 evolutionary models with stellar masses ranging from 0.530 to $0.741 M_{\odot}$. For each sequence of models, we computed the complete evolutionary stages of PG 1159 progenitors starting from the Zero Age Main Sequence. Evolution was pursued through the thermally pulsing AGB and born-again (VLTP) phases to the domain of the PG 1159 stars. The employment of such full evolutionary PG 1159 models constitutes a substantial improvement over previous studies on GW Vir stars regarding the stellar modelling.

Numerous detailed investigations of pulsating PG 1159 stars have been performed on the basis of artificial stellar models. In spite of the fact that significant pulsation damping and driving occur in PG 1159 envelope stars, the employment of such simplified stellar configurations appear not well justified in the case of these stars. This is in contrast to the situation of their more evolved counterparts, the white dwarf stars, for which their thermo-mechanical structure has relaxed to the correct one by the time the pulsational instability domains are reached. The main goal of the present work has been to assess to what degree the conclusions arrived at in previous studies on PG 1159 stars change when realistic stellar configurations are adopted.

Our study confirms the following results, already known from previous studies:

- $g$-modes in PG 1159 models are excited by the $\kappa$-mechanism due to partial ionization of carbon and oxygen. No abundance gradients between the driving region and the stellar surface are necessary to drive $g$-mode pulsations at the correct effective temperatures and period ranges.

- There exists a well-defined instability domain with a blue edge which is strongly dependent on the stellar mass.

- Different surface helium abundances lead to sizeable differences in the precise location of the theoretical blue edge of the instability domain.

- The instability domain splits into two separate regions, one of them at high luminosities characterized by long periods, 
and the other at low luminosities, corresponding to shorter periods, as recently demonstrated by GAS05.

- All pulsating PG 1159 stars lie in the predicted instability domain in the $\log \left(T_{\text {eff }}\right)-\log g$ plane.

- There is a very good agreement between the full period spectrum observed in GW Vir stars and the theoretical ranges of unstable periods.

- The pulsation periods of excited modes decrease with decreasing luminosity (increasing surface gravity), in line with the observational trend.

For our new findings, we mention:

- There exists a red edge of the instability domain in the high-luminosity (low-gravity) regime. This red edge is mass-dependent.

- The border of the instability domains in the $\log T_{\text {eff }}-$ $\log \Pi$ plane at the high-luminosity, long-period regime is well delineated.

- The pulsating PG 1159 Longmore 4 is located at the very red edge of the instability strip at high luminosities, a fact that could reflect the surprising behaviour observed in the spectral type of this star (Werner et al. 1992).

- Some non-variables occupying the instability strip have standard helium abundances and their presence between pulsators cannot be explained by the argument of Quirion et al. (2004).

- The pulsating nature and also the range of observed periods of PG 1153-035 - the prototype of the GW Vir class - are naturally accounted for by pulsationally unstable PG 1159 models with a stellar mass of $\sim 0.53-0.54 M_{\odot}$.

We found that generally the seismic masses (as inferred from the period spacings) are somewhat different to the spectroscopic masses, although the disagreement for the PG 1159-035 star is somewhat alleviated by our calculations. The persisting discrepancies could be attributed to a number of factors. On the observational side, possible systematic errors in the spectroscopic determination of $g$ and $T_{\text {eff }}$, and/or errors in the measurement of the period spacings of pulsating PG 1159 stars. On the other hand, differences in the microphysics or previous evolution may alter the location of the post-AGB tracks (Bloëcker 1995). It has been argued by Werner \& Herwig (2006) that the evolution during the TP-AGB (concerning third dredge-up efficiency and TP-AGB lifetimes) may be key in determining the location of post-AGB tracks. However, in preliminary simulations we have found that neither third dredge up efficiency nor TP-AGB lifetimes play an important role in determining the location of postAGB tracks. It remains to be seen if other physical assumptions like the overshooting efficiency during the core helium burning stage and early AGB (that also define the structure of the $\mathrm{C}-\mathrm{O}$ core and are completely free parameters) may play a role in the location of post-AGB tracks. We are currently performing simulations of full stellar evolution sequences under different assumptions to clarify these issues.

Acknowledgements. We wish to thank our anonymous referee for the constructive comments and suggestions that greatly improved the original version of the paper. This research was supported in part by the PIP 6521 grant from CONICET.

\section{References}

Alexander, D. R., \& Ferguson, J. W. 1994, ApJ, 437, 879

Althaus, L. G., Serenelli, A. M., Panei, J. A., et al. 2005, A\&A, 435, 631

Blöcker, T. 2001, Ap\&SS, 275, 1

Bond, H. E., \& Meakes, M. G. 1990, AJ, 100, 788

Bradley, P. A., \& Dziembowski, W. A. 1996, ApJ, 462, 376

Ciardullo, R., \& Bond, H. E. 1996, AJ, 111, 2332

Córsico, A. H., \& Althaus, L. G. 2005, A\&A, 439, L31

Córsico, A. H., \& Althaus, L. G. 2006, A\&A, 454, 863

Cox, A. N. 2003, ApJ, 585, 975

Dreizler, S., \& Heber, U. 1998, A\&A, 334, 618

Fujimoto, M. Y. 1977, PASJ, 29, 331

Gautschy, A. 1997, A\&A, 320, 811

Gautschy, A., Althaus, L. G., \& Saio, H. 2005, A\&A, 438, 1013 (GAS05)

Grauer, A. D., Bond, H. E., Liebert, J., Fleming, T. A., \& Green, R. F. 1987, ApJ, 323,271

Herwig, F. 2000, A\&A, 360, 952

Iglesias, C. A., \& Rogers, F. J. 1996, ApJ, 464, 943

Kawaler, S. D. 1988, ApJ, 334, 220

Kawaler, S. D., \& Bradley, P. A. 1994, ApJ, 427, 415

Kawaler, S. D., Winget, D. E., Hansen, C. J., \& Iben, I. 1986, ApJ, 306, L41

Kawaler, S. D., O'Brien, M. S., Clemens, J. C., et al. 1995, ApJ, 450, 350

Kawaler, S. D., Potter, E. M., Vuckovic, M., et al. 2004, A\&A, 428, 969

Koesterke, L., Dreizler, S., \& Rauch, T. 1998, A\&A, 330, 1041

Lee, U., \& Bradley, P. A. 1993, ApJ, 418, 855

Miksa, S., Deetjen, J. L., Dreizler, S., et al. 2002, A\&A, 389, 935

Miller Bertolami, M. M., \& Althaus, L. G. 2006, A\&A, 454, 845

Nagel, T., \& Werner, K. 2004, A\&A, 426, L45

O'Brien, M. S. 2000, ApJ, 532, 1078

O'Brien, M. S., Vauclair, G., Kawaler, S. D., et al. 1998, ApJ, 495, 458

Quirion, P. O., Fontaine, G., \& Brassard, P. 2004, ApJ, 610, 436

Reed, M. D., Kawaler, S. D., \& O'Brien, M. S. 2000, ApJ, 545, 429

Saio, H. 1996, Hydrogen Deficient Stars, ASP Conf. Ser., 96, 361

Schönberner, D. 1979, A\&A, 79, 108

Silvotti, R., Dreizler, S., Handler, G., \& Jiang, X. J. 1999, A\&A, 342, 745

Starrfield, S. G., Cox, A. N., Hodson, S. W., \& Pesnell, W. D. 1983, ApJ, 268, L27

Tassoul, M., Fontaine, G., \& Winget, D. E. 1990, ApJS, 72, 335

Unglaub, K., \& Bues, I. 2000, A\&A, 359, 1042

Unno, W., Osaki, Y., Ando, H., Saio, H., \& Shibahashi, H. 1989, Nonradial Oscillations of Stars (University of Tokyo Press), 2nd. edition

Vauclair, G., Moskalik, P., Pfeiffer, B., et al. 2002, A\&A, 381, 122

Vauclair, G., Solheim, J. E., \& Østensen, R. H. 2005, A\&A, 433, 1097

Werner, K., \& Herwig, F. 2006, PASP, 118, 183

Werner, K., Hammann, W.-R., Heber, U., et al. 1992, A\&A, 259, L69

Winget, D. E., Nather, R. E., Clemens, J. C., et al. 1991, ApJ, 378, 326 